\title{
Hazard Driven Threat Modelling for Cyber Physical Systems
}

\author{
Luca Maria Castiglione \\ Imperial College London \\ l.castiglione@imperial.ac.uk
}

\author{
Emil C. Lupu \\ Imperial College London \\ e.c.lupu@imperial.ac.uk
}

\begin{abstract}
Adversarial actors have shown their ability to infiltrate enterprise networks deployed around Cyber Physical Systems (CPSs) through social engineering, credential stealing and file-less infections. When inside, they can gain enough privileges to maliciously call legitimate APIs and apply unsafe control actions to degrade the system performance and undermine its safety. Our work lies at the intersection of security and safety, and aims to understand dependencies among security, reliability and safety in CPS/IoT. We present a methodology to perform hazard driven threat modelling and impact assessment in the context of CPSs. The process starts from the analysis of behavioural, functional and architectural models of the CPS. We then apply System Theoretic Process Analysis (STPA) on the functional model to highlight high-level abuse cases. We leverage a mapping between the architectural and the system theoretic (ST) models to enumerate those components whose impairment provides the attacker with enough privileges to tamper with or disrupt the data-flows. This enables us to find a causal connection between the attack surface (in the architectural model) and system level losses. We then link the behavioural and system theoretic representations of the CPS to quantify the impact of the attack. Using our methodology it is possible to compute a comprehensive attack graph of the known attack paths and to perform both a qualitative and quantitative impact assessment of the exploitation of vulnerabilities affecting target nodes. The framework and methodology are illustrated using a small scale example featuring a Communication Based Train Control (CBTC) system. Aspects regarding the scalability of our methodology and its application in real world scenarios are also considered. Finally, we discuss the possibility of using the results obtained to engineer both design time and real time defensive mechanisms.
\end{abstract}

\section{CCS CONCEPTS}

- Security and privacy $\rightarrow$ Distributed systems security; Information flow control.

\section{KEYWORDS}

Cyber Physical Systems Security, Threat Modelling, System Theoretic Analysis, Attack Graphs, Resilience

Permission to make digital or hard copies of all or part of this work for personal or classroom use is granted without fee provided that copies are not made or distributed for profit or commercial advantage and that copies bear this notice and the full citation on the first page. Copyrights for components of this work owned by others than ACM must be honored. Abstracting with credit is permitted. To copy otherwise, or republish, to post on servers or to redistribute to lists, requires prior specific permission and/or a fee. Request permissions from permissions@acm.org.

CPSIOTSEC'20, November 9, 2020, Virtual Event, USA

(c) 2020 Association for Computing Machinery.

ACM ISBN 978-1-4503-8087-4/20/11 . \$ \$15.00

https://doi.org/10.1145/3411498.3419967

\section{ACM Reference Format:}

Luca Maria Castiglione and Emil C. Lupu. 2020. Hazard Driven Threat Modelling for Cyber Physical Systems. In 2020 foint Workshop on CPS\&IoT Security and Privacy (CPSIOTSEC'20), November 9, 2020, Virtual Event, USA. ACM, New York, NY, USA, 12 pages. https://doi.org/10.1145/3411498.3419967

\section{INTRODUCTION}

Modern Cyber Physical Systems (CPS) feature several components operating together to monitor and control the surrounding environment. Disrupting the functionalities provided by CPSs impacts not only on business productivity but can also constitute a threat for operators' safety and to the environment in which they operate [1]. Critical National Infrastructures (CNI) such as healthcare, transportation networks, power grids, etc. are deployed as Cyber Physical Systems; the disruption of these activities impacts everyday tasks [4] [34] [33], state economies [20], and lives [24]. Cyber Physical Systems differ in many aspects from traditional software systems, and security problems must therefore be approached with a different perspective that also takes into account the evolution of the physical process. In CPSs attackers can leverage legitimate access points of the system (API) to disrupt the process and, in some cases, even damage the environment where it operates. Malicious actors have shown the ability to penetrate enterprise networks deployed around CPSs through a massive use of social engineering techniques and stolen credentials and to successfully infiltrate into the business level of an CNI [6]. Once inside the network, attackers can use legitimate system functionalities to disrupt the service provided or even damage physical components [20]. One example is the kill chain leading to the installation of the Crashoverride/Industroyer malware where cyber-criminals initially infiltrated the system using stolen credentials. Then, they navigated the IT network all the way to the Industrial Control System (ICS) and injected the payload leveraging legitimate channels and re-using credentials created during previous intrusions [34]. Adversarial actors can thus disrupt CPSs' activities by intruding their components and issuing commands when it is not safe to do so. In Model Based Engineering, System Theoretic tools such as System Theoretic Accident Model and Processes (STAMP) and System Theoretic Process Analysis (STPA) [21] [22][39] have been developed to address safety related issues at design level. These tools look at safety as an emergent propriety depending on a correct interaction between all the parts of the system. Hazards and consequent losses are caused by the unsafe application of control actions.

This work lies at the intersection between security and safety in the context of CPS. We introduce a methodology to perform hazard driven modelling of cyber and physical threats and impact assessment following an attack. We assume that the CPSs' architectural, functional and behavioural representations are available, along with the results of a hazard analysis. The latter typically includes a comprehensive list of hazardous scenarios that would 
result in losses with respect to business, operational environment and human life [9].

The proposed methodology starts with the analysis of behavioural, functional and architectural representations of the CPS and is organised in four steps, outlined in Figure 1. First (STEP 1.A), we apply System Theoretic Process Analysis (STPA) [22] on the functional model to elicit the existing causality links between commands issued and hazardous scenarios. The outcome of this step highlights the abuse cases [38]: collections of legitimate commands that the attacker might aim to use to trigger hazards. During the second step, we perform two mappings (STEPS 2.A and 2.B). One links together the functional and architectural models of the CPS, while the second is constructed between the functional and the behavioural models. From an adversarial perspective, this is a two stage process aming to, firstly, assign a semantic meaning to data-flows defined within the System Theoretic Model and, secondly, to link the attack surface with the data-flows. The objective is to understand the impact of adversarial actions on the evolution of the controlled physical process.

We then proceed with the enumeration of those components (Target Nodes) in the architectural representation, whose impairment provides the attacker with enough privileges to alter or disrupt the information data-flows (STEP 3.A). Applying this methodology leads to three outcomes: we compute a comprehensive attack graph (AG) of all exploitation paths leading to compromise of the Target Nodes (STEP 4.C), we provide a qualitative (STEP 4.B) and then a quantitative (STEP 4.A) impact assessment of compromising on one or more Target Nodes. We illustrate the methodology by applying it to a small scale example of a Communication Based Train Control (CBTC) system. We further discuss aspects regarding the scalability of our methodology and its application to real world scenarios.

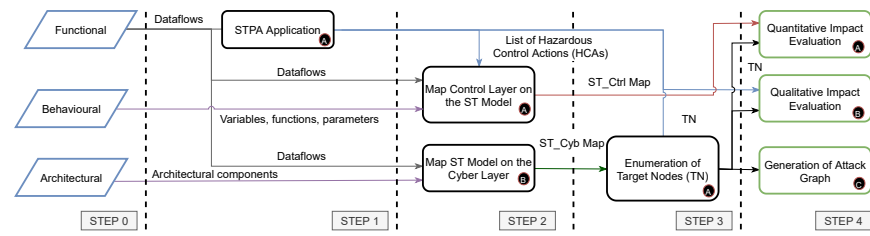

Figure 1: Hazard Driven generation of Attack Graph for $\mathrm{Cy}$ ber Physical Systems.

The contributions of our work are as follows:

- We propose a mapping between the architectural and functional models of a CPS

- We propose a mapping between the behavioural and functional models of a CPS.

- We enumerate the architectural components that are potential targets of threats, given a high-level hazard analysis. We leverage the target list, along with the system architectural model, to compute an Attack Graph for the CPS that comprises all known exploitation paths enabling an attacker to compromise emerging system properties such safety and domain specific performance.

- We propose both a qualitative and quantitative assessment of the impact of cyber and physical attacks against CPSs.
We apply our methodology to a small scale example featuring a Communication Based Train Control System (CBTC). Aspects regarding the scalability to a real world setting are discussed. The results we obtain are analysed aiming to engineer defensive strategies both at design time and at run time.

Our work is organised as follows: in Section 2 we give an overview of the related work, in Sections 3 and 4 we introduce the models used to represent different aspects of a CPS and how these representations relate to each other. In Section 5 we introduce a hazard driven threat modelling methodology that combines the three representations. The framework presented is applied on a use case in Section 6, while in Section 7 we explore further the use of our model to assess the impact of attacks against CPSs.

\section{RELATED WORKS}

The resilience of Cyber Physical Systems (CPSs) to attacks is addressed in literature from several perspectives most of which are based in either Control or Systems Theory. Research based in Systems Theory, such as Systems-Theoretic Accident Modelling and Processes (STAMP) [21], provides a functional view of the CPS and the interactions that its components have one with each other. System Theoretic Process Analysis (STPA) [21][22] is a hazard analysis methodology applied on a system theoretic model and is built on the top of STAMP. STPA sees safety as an emergent property and is based on the assumption that incidents are caused by unsafe applications of control actions. It therefore investigates causalities in complex systems through the analysis of the information flow. STPA has also been applied to security aspects leading to STPA-Sec [39],[28]. STPA-Sec starts from a list of system hazards and aims to find the security violations that enable attackers to unsafely apply control actions. In these models, security aspects are treated with respect to safety and the methodology does account for targets other than critical functionalities [12]. These two limitations can be overcome by introducing at the requirements modelling stage, broader hazardous scenarios that also take into account non-safety related losses.

STPA is a well defined and structured process and studies such as [31] [28], [39] and [12] successfully find adversarial hazardous scenarios. However, STPA does not provide a systematic way to to quantify and summarise the results of the analysis and results obtained from the application of System Theoretic approaches are mostly qualitative as they are derived from the study of a functional model and are therefore not sufficient to perform attack detection and response. Furthermore, the methodologies used do not integrate well with the design and implementation frameworks commonly used in industrial environments. They are applied through dedicated and non-automated processes and do not easily adapt to systems of high dimensionality. A substantial missing step is relating such solutions to the architectural level. A contribution, in this sense, is given in [16], which successfully models control dependencies at functional level and eventually validates the results on a deployed architecture, spanning from the functional to the architectural models of the CPS analysed.

Control theoretic approaches leverage the behavioural model of the system, including its internal dynamics and algorithms, to 
evaluate the impact of an attack on the controlled process. Research presented in [3], [10], [36] uses control theory to elaborate anomaly detection algorithms. In their frameworks, the attacker actions map directly to the equations describing the system dynamics and control algorithms [36] [25]. The authors of [19] build a restricted model for actuators to constrain attacker actions, while in [14] the authors leverage Moving Target Defence (MTD) techniques to respond to attacks on vehicle networks. Often studies leveraging control theory for resilience purposes suffer from two limitations. Firstly, they usually assume the absence of constraints (e.g., safety constraints) when developing countermeasures [13] to attacks. Secondly, the architectural aspects of the CPS are not taken into account. In other words, the effects of the attack are mapped on the signal while it is not clear which vulnerabilities the attacker needs to exploit in order to obtain privileges to alter the information.

Our work aims to overcome the limitations of System Theoretic and Control Theoretic approaches by introducing a methodology that is orthogonal with respect to the models of the CPS, exploiting the information they provide from different angles. In particular, we leverage a map between the architecture and the System Theoretic Model to identify possible targets (Target Nodes) for an attacker whose aim is to impair system safety or performance. Thus, we analyse the solutions found (Target Nodes), mapping on the Control Model the effect of the attack. This enables us to understand qualitative aspects of an attack (possible hazardous scenarios) but also to quantify its effects and the recovery strategies in a way that is coherent with both the requirements of the Control Model and with the physical constraints imposed by the Architecture.

Authors in [17] present an interesting study of demand manipulation attacks against a power grid, discussing various resilience aspects across different levels of the system. While their work is centred around a specific use case and scenario, we aim to propose a general methodology to perform a hazard driven security analysis of a CPS. In [5], the authors use a systemic approach to analyse code on Programmable Logic Controllers (PLC) and find interaction between Attack Points and Controlled Infrastructure, assuming the availability of the source code for the analysis. The dimension and quality of their results is also highly correlated with the quality of the code. Moreover, the data-flow graph generated is large and grows significantly with the complexity of the CPS. In contrast, our work provides a methodology that is agnostic to specific implementations. It provides a hazard driven analysis rather than a simple enumeration and starts from an analysis of the hazards. However, we require for this the availability of architectural, control and system theoretic models.

In [32] attack trees are used to quantify the risk to embedded vehicular networks. High level attack goals are derived as abuse cases from safety requirements that are specified during the design phase with expert knowledge. The work is further extended in [23], which maps the steps needed to carry out an attack to the actual topology of the on-board network. The methodology introduced in our paper differs in many aspects from the work presented in [23]. Firstly, we systematically derive high level attack goals by applying STPA to the functional model of the CPS. Secondly, while [23], leverages the attack tree model from [32], we use attack graphs instead. Here a set of nodes (Target Nodes) describes the post-exploitation privileges that the attacker needs to obtain to compromise a particular data-flow, which is identified in previous steps by applying STPA. In other words, critical data-flows are identified with STPA and we compute an attack graph comprising the paths that lead the attacker to obtain enough privileges to compromise them. In doing so we build a comprehensive attack graph for the system considering all the Target Nodes and avoiding redundant description of common paths leading to different high-level objectivse. Finally, by relating the architecture of the CPS with its behavioural model we potentially enable to compute solutions with respect to cost functions defined on on system performance.

\section{SYSTEM MODELS}

Our representation covers three different aspects of a CPS. It is a variation of that proposed by Hahn et al. in [15] and Ericson et al. in [9] and consists of 3 models, as shown in Figure 2: Functional, Architectural and Behavioural.

Before going further, we introduce the System Under Test (SUT) which will serve as use case to illustrate the application of our methodology. It consists of a Communication Based Train Control System (CBTC) featuring $N$ convoys, that is part of a transportation network. Each train is equipped with on-board sensing, communication and control units and autonomously travels on its track. Convoys run at the maximum allowed speed to maximise the throughput of the infrastructure, while always keeping a predefined safe distance between themselves and preceding trains. This is a distributed control system as convoys take control decisions individually using the information received from their own sensors and other trains. We also account for the presence of a zone controller (supervisor) whose aim is to manage the traffic by imposing speed limits to convoys, depending on their position on the path. The following losses have been identified, from expert knowledge: (L1) Loss of human lives, (L2) Injuries, (L3) Severe damages to instrumentation, (L4) Damages to the image of the company, (L5) Minor damages to instrumentation. Subsequently, the following hazards have been identified, also by using expert knowledge: (H1) Collision (between two or more convoys), (H2) Derailment, (H3) Disruption of the service. In particular, if hazards H1 and H2 manifest, the system faces losses of the type L1, L2 and L3, while $\mathbf{H 3}$ is only connected to $\mathbf{L} \mathbf{4}$.

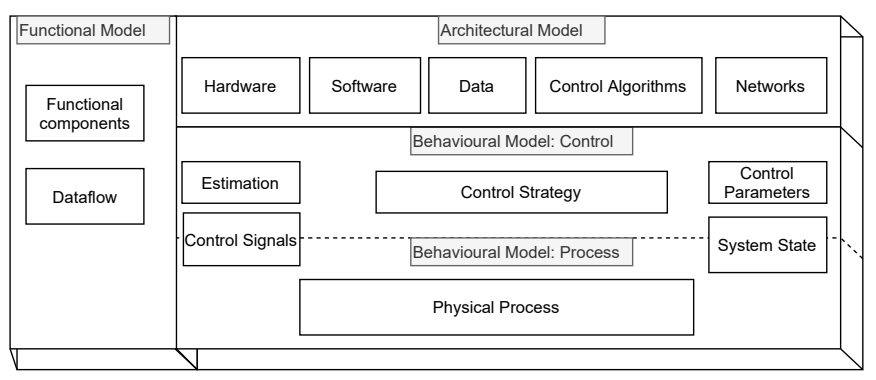

Figure 2: Representation of a Cyber Physical System based on the models proposed in [15] and [9]. 


\subsection{Architectural Model}

The architectural model consists of hardware and software entities that shape the architecture of the CPS. In order to represent different aspects of a layer comprising both hardware and software components, it is usually modelled using an Architecture Description Language (ADL). In this sense, the ADL provides statements to describe hardware devices as well as software routines, data-flow connections and access to networks and buses at different levels of detail. With this in mind, we chose a subset of Architecture Analysis Design Language (AADL) [11] to model the SUT. Among the AADL components we recall Process, Data, Subprograms, Memory, Devices, Bus. Processes represent the addressing space to execute subprograms that consume data, while devices represent sensor and actuating units interacting with the system. AADL components communicate with each other over buses while connections are defined through ports, which enables the exchange of events or raw data. AADL also introduces the concept of flows; these group together data exchanges, enabling the specification of high level data-flows across multiple architectural components, from their source to their sink. This characteristic is particularly useful when establishing a mapping between the architectural and the system theoretic models of the CPS. Since this model represents the deployment of the system, it is also representative of the Attack Surface exploitable by cyber (or physical) criminals.

\subsection{Behavioural Model}

The behavioural model of the CPS comprises two layers, the Control and the Physical Layer respectively, as shown in Figure 2. We use an analytic representation to describe the evolution of the system in time and the applied control strategies.

The Control Layer includes the control algorithms that run on the system's controllers to regulate the Physical Process. Outputs from the controllers (or control actions) are usually expressed in the form $u(t, x(t))$ where $x(t)$ is the system state observed on the basis of sensors' output (this is the case of a state-feedback control (SFC)) and $t$ is the time. Alternatively, $u(t, y(t))$ where $y(t)$ is the output (direct measurements) made on the Physical System. Our use case includes two hierarchical controllers on each train: the external loop works in a distributed fashion aiming to synchronise the train with the preceding one. In Equation 1 we show the control law regulating the internal loop of $\operatorname{Train}_{i}$. The controller output $u_{i}^{i n t}$ is a signal which can assume real negative (braking action) or positive (throttling) values.

$$
u_{i}^{i n t}(t)=-K_{P}\left(y_{i}(t)-\hat{y}_{i}\right)-K_{I} \int_{0}^{t}\left(y_{i}(\tau)-\hat{y}_{i}\right) d \tau
$$

The control law is proportional integral action on train speed $y(t)$ and the control objective is $y_{i}(t)=\hat{y}_{i}$. The value $\hat{y}_{i}$ is calculated from the external controller, whose dynamics is shown in equation 2 , as $\hat{y}_{i}=u_{i}^{\text {ext }}$.

$$
u_{i}^{\text {ext }}(t)=\left\{\begin{array}{lc}
-K_{P}\left(p_{i}(t)-p_{i-1}(t)-\hat{p}\right) & \dot{p}_{i} \leq s_{L} \\
s_{L} & \text { otherwise }
\end{array}\right.
$$

This is a proportional action on the position of the $\operatorname{Train}_{i}$ and the control objective is $p_{i}(t)-p_{i-1}(t)=\hat{p}$ with $p_{i}, p_{i-1}$ being respectively positions of $\operatorname{Train}_{i}$ and $\operatorname{Train}_{i-1}$ while $\hat{p}$ is a safe distance that must be kept between the two convoys. The control output is saturated from the speed limit $s_{L}$; we make the realistic assumption that $s_{L}$ is dimensioned such that the control objective is reachable. To achieve the control objective $p_{i}(t)-p_{i-1}(t)=\hat{p}$, we assume that the internal controller is much faster than the external one. In other words, the inner system reacts almost instantaneously to the external control.

The Physical Layer consists of the description of the behaviour of the Physical Process. It can be described in the affine form as:

$$
\left\{\begin{array}{l}
\dot{x}=f(x, \mu)+g(x) u \\
y=h(x)
\end{array}\right.
$$

where $f$ is the system dynamics, $x(t)$ is the state and $\mu$ a vector of system parameters. Then, $h(x)$ is the function that, applied to the state, returns the output $y(t)$ while $g(x)$ provides information about how the output of the controller influences the system state. As the aim of our work is to model the security aspects of complex systems, we used a simple mass-damping equation to model the dynamics of the moving convoys. This is described by Equation 4 as:

$$
\left\{\begin{array}{l}
\dot{x}=y \\
\dot{y}=-\frac{b}{m}+\frac{1}{m} u
\end{array}\right.
$$

with $x, y$ being respectively the position and speed of $\operatorname{Train}_{i}$ and $b, m$ its dumping coefficient and mass. $u=u_{i}^{i n t}$ is the applied force. For the sake of brevity, we omitted subscript $i$, as variables and parameters in equation 4 all are particularised to $i-t h$ convoy.

\subsection{Functional Model and Analysis}

We use System Theoretic Accident Model and Processes (STAMP) and System Theoretic Process Analysis (STPA) to model the functional aspects of the system. We also augment the System Theoretic Model by explicitly defining relationships between the input and the output of system components. STAMP is a causality model that studies safety as an emergent property of distributed systems[21] [18]. The System Theoretic (ST) Model is a 3-tuple $\mathcal{S T}=(C, F, K)$. In particular, $C$ is the set of Components of the CPS. They are represented through functional blocks interacting with each other. Components are named using the syntax

$$
\left(o_{1}, o_{2}, \ldots, o_{M}\right) \leftarrow \operatorname{cid}: \operatorname{name}\left(i_{1}, i_{2}, \ldots, i_{N}\right)\left[r 1, r_{2}, \ldots, r_{K}\right]
$$

Where $o_{1}, o_{2}, \ldots, o_{M}$ are the outputs, $\left(i_{1}, i_{2}, \ldots, i_{N}\right)$ are its inputs and $\left[r 1, r_{2}, \ldots, r_{K}\right]$ are the role/roles assumed by the component. Roles provide the privilege of triggering (calling) a control action. cid is a unique identifier for the component in the system while name is its symbolic name. $K$ is the set of subsystems that group functional components $\left(c_{i} \in C, \quad i=1, \ldots, N\right)$ together, according to a predefined logic aiming to provide a compartmentalised and more efficient representation. Finally, $F \subseteq C \times C$ is the set of data-flows in the CPS. They represent the exchange of information between components and are characterised by a source and a destination. They are sketched as arrows in the ST model and their direction points to the destination of the exchange. We distinguish between Control Actions and Feedback. Namely, a Control Action $d_{c a} \in C A \subseteq$ $F$ is defined as:

$$
\text { id : name }\left[r 1, r_{2}, \ldots, r_{K}\right]
$$


where $i d$ is the unique identifier, name is its name and $\left[r 1, r_{2}, \ldots, r_{K}\right]$ are the privileges required to trigger (call) it. The latter coincide, in our model, with the cid of components that trigger them. Similarly, a Feedback $d_{f} \in F B \subseteq F$ is defined as:

$$
i d: \text { name }\left[r 1, r_{2}, \ldots, r_{K}\right]
$$

where $\left[r 1, r_{2}, \ldots, r_{K}\right]$ are the privileges required to write the feedback. Composite structures featuring feedback loops, hierarchies and distributed control patterns are created by leveraging several data-flows to regulate complex interactions. Note that the components in this representation are functional, i.e., they represent the application of transformations to the data-flow in the CPS.

Finally, we also introduce the precedence relationship introduced by [35]. Namely, $d_{0}$ is defined as output of $c_{1}$, which takes $d_{0}$ and $d_{1}$ as inputs. We say that a precedence relationship exists between the $d_{2}$ and $\left(d_{0}, d_{1}\right)$, therefore the health of $d_{2}$ depends on the health of $d_{0}$ and $d_{1}$. This is also expressed in the form:

$$
d_{2} \leftarrow d_{0}, d_{1}
$$

We call the degree of the precedence relationships the distance between two data-flows. For example, we say that there exists a precedence relationship of degree 2 between $d_{0}$ and $d_{3}$ and $d_{0}$ and $d_{4}$ if and only if:

$$
d_{0} \leftarrow\left(d 1, d_{2}\right) \quad d_{1} \leftarrow\left(d 3, d_{4}\right)
$$

In Figure 3 we show the System Theoretic Model for the System

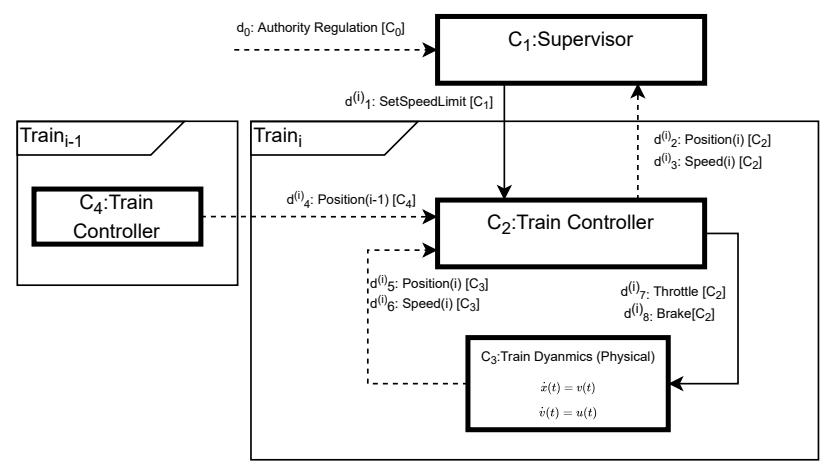

Figure 3: System Theoretic Model of the Train System. Feedback data is referred by dashed arrows while filled ones point to Control Actions.

Under Test ( $N=2$ trains). It comprises 4 functional blocks representing, namely, the Remote Operator $\left(C_{1}\right)$, the Controller of the $\operatorname{Train}_{i}\left(C_{2}\right)$ and the Physical Process enclosing the train dynamics $\left(C_{3}\right)$. We show $\left(C_{4}\right)$, the Controller of the leading Train. Of the 8 data-flows in the System Theoretic Model, 3 are Control Actions and 5 are Feedback. In particular $d_{1}^{(i)}$ and $d_{7}^{(i)}, d_{8}^{(i)}$ respectively enclose the control actions SetSpeedLimit (event) and the Throttling and Braking action (continuous control signal). On the other hand, the remaining arrows express feedback from Sensors $\left(d_{5}^{(i)}, d_{6}^{(i)}\right)$ and Controllers $\left(d_{3}^{(i)}, d_{4}^{(i)}\right.$ and $\left.d_{7}^{(i)}\right)$. The $i$ exponent is used to distinguish two different data-flows transporting semantically similar

\begin{tabular}{lcccccc}
\hline CA & A NR & NA R & \multicolumn{2}{c}{ WT } & \multicolumn{2}{c}{ WD } \\
& & & SE & SL & TS & TL \\
\hline Brake & H3 & H1, H2 & H3 & H1, H2 & H1, H2 & H3 \\
Throttle & H1,H2 & H3 & - & H3 & H3 & H1 \\
Speed Limit & H3 & H2 & - & - & - & - \\
\hline
\end{tabular}

Table 1: Results of the STPA analysis on Unsafe Control Actions (UCAs) carried on the train systems. Control Actions are specified in the form Component:Control Action. UCAs are analysed in the following four cases as specified by Leveson et al. : Applied and Not Required (A NR), Not Applied and Required (NA R), Wrong Timing (WT) - Starts Early (SE), Starts Late (SL) - and Wrong Duration - Too Short (TS), Too Long (TL).

pieces of information in the CPS (e.g., $d_{2}^{(i)}$ is Position of Train $i$ while $d_{2}^{(i+1)}$ is the Position of $(i+1)-t h$ Train).

System Theoretic Process Analysis (STPA) [21] is a framework built on the top of STAMP and enables causal safety analysis on functional block diagrams (STAMP models). In STPA, an incident is the outcome of one or more unsafe applications of control actions [21]. Control actions (CAs) become unsafe (UCAs) if one of the following four cases applies: $\mathrm{CA}$ is required but not applied, $\mathrm{CA}$ is applied and not required, $\mathrm{CA}$ is applied with a wrong timing (before, after, wrong switching sequence) or applied with the wrong duration (too long or too short). Unsafe Control Actions (UCAs) are issued when the Controller has a view of the process that is, to an extent, incomplete or incorrect. This can happen because of failures in the controller or in the sensing input. The whole idea of STPA consists of enforcing safety constraints aiming to avoid the occurrence of unsafe control actions and consequent losses. The process is driven by expert knowledge and it has been semi-automated in [37]. It starts with the identification of the hazards and losses that may affect the system under analysis. First, given a list of control actions (CA), their unsafe application is explored with respect to hazards that can be caused. Then it proceeds with an analysis of the feedback for UCAs to track where, in the system, safety conditions are broken and where to reinforce weaknesses. In [39] Leveson and Young present STPA-Sec, consisting in the application of STPA with respect to system security.

In the case of the CBTC system (Figure 3), unsafe applications of Throttle and Brake control actions can lead to hazards H1, H2 and $\mathbf{H} 3$ while the unsafe application of SetSpeedLimit potentially causes hazardous scenarios $\mathbf{H} 2$ and H3. We highlight that, since SetSpeedLimit is an discrete action, Wrong Duration and Timing cases do not apply. A list of hazardous control actions is summarised in Table 1.

We introduced three representations to model different aspects of a CPS. We employ a System Theoretic model to describe functional aspects of the CPS while its architecture is outlined through a subset of AADL. The evolution of the system is described by a behavioural model through differential equations; we distinguish between the control and the physical layer, enclosing equations representative of the controller and the physical process respectively. 


\section{MAPPING THE MODELS}

In this Section, we perform a mapping between the System Theoretic (functional) model of a CPS and its architectural and behavioural representations. In Section 4.1, we bind control functions, variables and parameters to the data-flow and components of the System Theoretic model. This enables us to highlight the importance of the role that elements of the functional model play with respect to the evolution of system performance ${ }^{1}$. In Section 4.2, a second map is built between functional and architectural models to investigate how data-flows actually run through the architectural stack. In security settings, the latter provides a link between the System Theoretic Model and the attack surface.

\subsection{From System Theoretic to Behavioural Model}

The aim of this Section is to explain the information data-flow in the System Theoretic model with respect to control functions, variables and parameters defined within the behavioural representation of the CPS Architecture.

The process follows the research introduced by Thomas et al. in [37]. In fact, [37] provides a semi-automatic mapping between safety requirements and the system variables and parameters. In a similar way, we now link these latter to their counterpart in the behavioural model. This process is done at design level and its complexity grows with the complexity of the CPS for heterogeneous systems. However, the mapping can be semi-automatised in homogeneous (e.g. multi-agent) systems such as vehicle networks, racks, etc..

We distinguished between 2 types of data-flows: Control Actions and Feedback. CAs flow between two Controllers or a Controller and the respective controlled process through an Actuator. In the first case, they can be streams or events, while in the second case they are mostly streams. We distinguish between three types of Control Actions:

- Reference: Imposed either by a human or by an algorithmic supervisor. If the controller solves a regulation problem, the control action is an event, imposing the new reference to follow. On the other hand, in a Tracking scenario, it is a stream transporting the signal to follow. [Source: Higher Level Controller/Supervisor. Destination: Controller].

- Parameter: Imposed either by a human or by an algorithmic supervisor. It is usually an event when updated by an operator. It can also be a stream, in case of adaptive systems. [Source: Higher Level Controller/Supervisor. Destination: Controller]

- Output of control algorithm: Imposed by a controller, it can be an event if the destination is a lower level controller (i.e. 'open/close') or a stream if the destination is an actuator (i.e. numeric signal, DAC) [Source: Controller. Destination: Lower Level Controller or Physical Process/Actuator]

We distinguish between two types of Feedback information dataflows:

\footnotetext{
${ }^{1}$ The evolution in time of signals that are the output of system components. Individual outputs of system components as well as aggregate measurements can be used to evaluate performance, depending on the application domain of the CPS.
}

- Process output: Sent by a sensor, it is an event such as the code of a state (i.e. '0: opened /1: closed') if the sensing unit embeds a lower level controller. It can also be a numeric signal as a stream (i.e. ADC), in the case of simpler sensors. [Source: Sensor/Controller. Destination: Controller]

- External process output: Sent by the Controller of another Physical Process in the system. This is useful in distributed patterns where the controller needs to synchronise the state of the regulated process with the state of another process, received from the respective controller [Source: Controller. Destination: Controller].

With respect to the System Theoretic Model shown in Figure 3, given the behaviour defined in Equations 1 and 2 (Section 3), we propose the following mapping. This can be synthesised by hand or in a semi-automatic way, at scale, if a set of rules is provided along with the system design. Although expert knowledge is always required to link one or more data-flows to control elements, we believe that this operation does not introduce further overhead to the design process. In fact, this kind of mapping is already being carried out to guarantee the traceability of safety requirements across the CPS.

- $d_{1}^{i}$ (Reference - Event Control Action) maps to $s_{L}$, speed limit and saturation for the external controller $u_{i}^{\text {ext }}$.

- $d_{7}^{i}$ (Output of Control Algorithm - Stream Control Action) maps to $u_{i}^{\text {ext }}>0$ and is the positive force pushing the $i-t h$ train forward.

- $d_{8}^{i}$ (Output of Control Algorithm - Stream Control Action) maps to $u_{i}^{\text {ext }} \leq 0$ and is the negative force braking the $i-t h$ train.

- $d_{2}^{i}$ (Output - Stream Feedback) maps to $p_{i}(t)$ and is the position of the $i-t h$ train sent from the controller to a higher level controller (supervisor).

- $d_{3}^{i}$ (Output - Stream Feedback) maps to $\dot{p}_{i}(t)$ and is the speed of the $i-t h$ train sent from the controller to a higher level controller (supervisor).

- $d_{5}^{i}$ (Process Output - Stream Feedback) maps to $p_{i}(t)$ and is the speed of the $i-t h$ train sent from the position sensor to the $i-t h$ train controller.

- $d_{6}^{i}$ (Output - Stream Feedback) maps to $\dot{p}_{i}(t)$ and is the speed of the $i-t h$ train sent from the position speed sensor to the $i-t h$ train controller.

- $d_{4}^{i}$ (Output - Stream Feedback) maps to $p_{i-1}(t)$ and is the speed of the $(i-1)-t h$ train sent from the controller to the controller of $i-t h$ train.

\subsection{From Architectural to System Theoretic Model}

The architectural model of the CPS is representative of the deployed architecture and it is described using a subset of an AADL, extended with a security annex that enables the explicit specification of roles and permissions. The System Theoretic (ST) Model is a 3-tuple $\mathcal{S T}=(C, F, K)$ with $C$ set of functional components, $F$ set of dataflows and $K$ set of subsystems. We use flow statements [11] to describe the data-flows between the architectural components (or nodes) of a system, as shown in Figure 4. The following notation states that the architectural component $\theta$ handles the data-flow 
$d \in F$

$$
\theta \in \Phi(d)
$$

where $\theta$ can be a any element of the AADL subset we defined in the previous Section (device, system, program, bus, network, etc.). Nodes access the information data-flow with read or write permissions, consistently with the operations that they need to perform. The following notation is used to state that component $\theta$ accesses the i data-flow $d \in F$ with reading privileges

$$
\theta \in \Phi(d, " r ")
$$

we replace " $r$ " with " $w$ " if $\theta$ accesses $d$ with writing privileges, instead.

In Figure 4 we show the mapping between System Theoretic and architectural models. Thick arrows provide a colour coded representation information data-flow across AADL systems and devices. Position and Speed Sensors are represented by AADL devices while the Train Controller is a system enclosing the process representing the control algorithm (omitted for the sake of brevity). Sensors are the source for data-flows $d_{5}^{i}, d_{6}^{i}$ and communicate with Controller (destination of $d_{5}^{i}, d_{6}^{i}$ ) through the Canbus. The Controller receives the speed limit and the position of the preceding train from the Zone Controller $\left(d_{1}^{i}, d_{4}^{i}\right)$ over a radio connection. The data-flow transporting the control action Speed Limit originates from the business layer and travels across the whole system.

Finally, we formally define the set of Target Nodes for a control action. In particular $T N(d), \quad d \in F$ are the components with read and (or, depending on the requirements) write privileges on $d$. For example, the set $T N\left(d_{7}^{i}\right)=\{$ Controller, Canbus, Actuator $\}$.

We linked together functional, architectural and behavioural models of a CPS. Although for the purposes of this work, the mapping has been built manually, parts of it could be automated and the process does not add significant overhead to the design, implementation and deployment phases already used in industrial settings. To this extent, in Section 4.2, we related STPA and AADL through flow statements. Such flow modelling across AADL components is a powerful strategy already used in literature [11] [26] [8] to highlight how the information data-flow is handled across the architectural stack. Furthermore, in Section 4.1 we linked control variables, parameters and functions of the behavioural model to the information data-flow. This is also a common practice to trace safety requirements, among others, across different stages of industrial processes.

\section{THREAT MODELLING}

In this Section, we introduce a hazard driven Threat Modelling process to evaluate the impact on the system's emergent properties ${ }^{2}$, following the exploitation of both cyber and physical threats. The difficulty intrinsic in this modelling activity lies in its span across domains. In particular, attack objectives are defined within the system theoretic model while the attack surface is defined within the architectural model. We leverage the mapping performed in Section 4.2 to relate hazardous scenarios and respective losses to the exploitation of a subset of system vulnerabilities and weaknesses of architectural components. Furthermore, we introduce Logical Attack Graphs [30][29] in Section 5.3 to highlight the exploitation

\footnotetext{
${ }^{2}$ Domain specific emergent behaviours, including Safety [22].
}

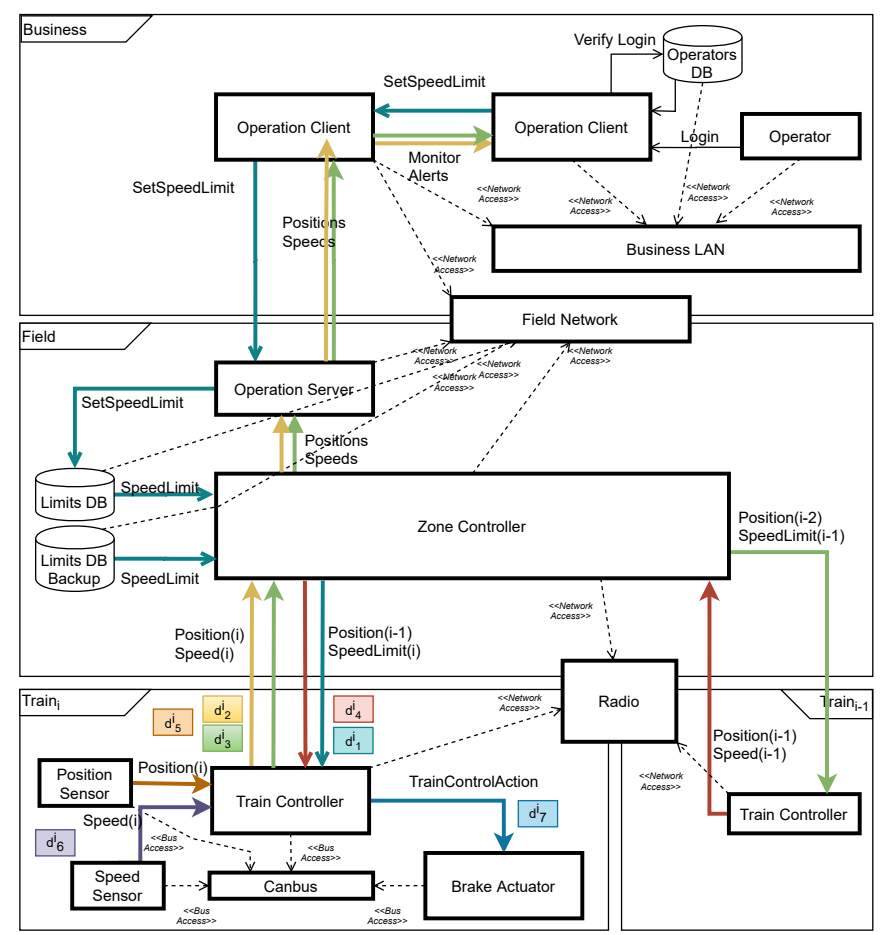

Figure 4: Illustration of the map between the architectural model of the Cyber Physical System and its functional model. Thick arrows provide a colour coded representation information data-flow across AADL systems and devices.

paths that an attacker can follow across the architecture of the CPS. The proposed threat model activity comprises three main steps: (1) Definition of attacker capabilities, scope and high level objectives of the attack. (2) Given assumptions made at the previous stage, we enumerate components of the architectural model which constitute a potential target of a cyber (or physical) attack. (3) We make use of the results gathered from the enumeration at previous step to compute the Attack Graph for the high level objectives. Finally, in this work we make the important assumption that components of the CPS interact in a safe manner. Therefore, we exclude safety violations and disruptions caused by failures. In fact, while it is true that safety-critical CPSs are inherently designed to be resilient to unsafe conditions, a successful attack can (1) Trigger fail safe mechanisms resulting in a disruption of availability. (2) Actively target safety protections [7]. (3) Bypass safety protections by tampering with the representation that the controller has of the physical process and actively call control actions when it is unsafe to do so [20]. .

\subsection{Model elements}

In this Section, we provide an overview on the main elements characterising the proposed threat modelling activity.

Adversaries: The threat model we propose can account for several adversarial actors. We make the general assumption that adversaries have a white box knowledge of system architecture, functional 
and behavioural models. We also assume that existing weaknesses and vulnerabilities are known. This assumption provides the defender with an overview on all the exploitation paths that attackers can use. The attack starts from a pre-defined injection-point and ends at a Target Node which defines a potential objective. In fact, through the exploitation of weaknesses or vulnerabilities affecting one of the Target Nodes, attackers can achieve high level objectives such as the abuse of functionalities, potentially triggering hazardous scenarios that result in system level losses. The hypothesis on the adversary's capabilities can easily be reduced to fit different offensive scenarios. In this sense, a reduced knowledge of architecture, topology and vulnerabilities or offensive capabilities coincides with pruning the Attack Graph leading to Target Nodes. On the other hand, we do not consider a node as a potential attack target if the attacker has no interests in reaching it. The latter coincides with limiting the attacker's knowledge of functional and behavioural models.

Scope: Within the threat modelling activity, we account for both cyber and physical attacks against system components. This includes analogical channels that sensors and actuators use to interact with the Physical Process. This is done by including in the Attack Graph, besides classical cyber vulnerabilities ${ }^{3}$, weaknesses that are intrinsic to CPS's deployment such as, for instance, the susceptibility of a sensor to the intentional exposure to heat.

Objectives: With reference to [15], we distinguish between two categories of high level objectives: Disruptive action: refers to the intentional disruption of system functionalities. Attacks of this kind are usually detected based on domain-specific measurements. In more sophisticated cases, disruptive actions can aim to destroy system components [20] or to waste defenders' resources, making the system work in sub-optimal conditions. In order to cause a disruptive action, an attacker needs to exploit one or more abuse cases, defined at system theoretic level. Mission hijack: refers to an attacker aiming to hijack the scope of the mission by changing the behaviour of the CPS.An example of this is the attack against a supply chain presented in [2]. In order to achieve this objective, attackers need a wide knowledge of the functional and behavioural models of the CPS to identify the minimum subset of information data-flows they need to control.

Attack Modalities: We consider three high-level attack modalities that an adversary can leverage to directly or indirectly apply unsafe control actions. The first modality describes direct attacks against a Control Action. In this case, the attacker gains enough privileges to be able to directly tamper with or disrupt the information data-flow transporting the control action itself; an example would be the exploitation of Target Nodes, such as actuators, buses or field networks. This case applies to both Stream and Event Control Actions. Another scenario, similar to the first one, sees the attacker exploiting vulnerabilities on Target Nodes such as Human Machine Interfaces (HMI) and Controllers, earning privileges to arbitrarily call legitimate low-level APIs. This is the case of an attack aimed to obtain privileges to apply a Event Control Action. Finally, the third scenario considers indirect attacks against Control Actions and Feedbacks; an example of this attacks is given by threats against Target Nodes handling information data-flow, that is in a precedence

${ }^{3}$ Learnt by running a vulnerability scanning operation on the network. relationship of any degree with the targetted Control Action. This last scenario applies to both Control Actions and Feedback.

Outcome: The outcome of the threat modelling is twofold. On one hand, we provide an enumeration of potential targets as Target Nodes. We also compute an attack graph for the CPS, comprising the attack paths towards Target Nodes. In other words, we aim to explain the consequences that a successful attack against part of the Cyber Physical System architecture has on its behaviour.

\subsection{Target Enumeration}

Following the definitions provided about attacker capabilities, scope and high level objectives, the proposed threat modelling activity proceeds with the enumeration of Target Nodes; the components that are ultimately targeted to achieve a high-level objective.

The enumeration process starts from the definition of a high level objective between Disruption of a Service and Mission Hijacking. In the first case, we analyse the results of the System Theoretic Process Analysis (Table 1, Section 3) to identify Unsafe Control Actions that, if triggered, can lead to the hazardous scenarios of interest. The following step consists of identifying, within the System Theoretic Model, information data-flows that can be of interest for an attacker. The identification is carried out according to the different attack modalities. Therefore, the output at this step is a list of data-flows including the ones transporting the control action and those which are in a precedence relationships of any degree with the latter. On the other hand, in case of an attacker aiming to hijack the objective of the mission, a wide knowledge of the CPS behavioural and functional models is required. This latter finds the minimum subset of control and system variables that needs to be accessed (in reading or writing mode) to shift the behaviour of the underlying Physical Process. Given the elements of the behavioural model that the attacker needs to access, it is possible to leverage the mapping with the system theoretic model to identify the information data-flows that can be of interest for an attacker. Successively, the mapping between architectural and system theoretic model found in 4.2 is leveraged to trace potential targets within the architectural deployment given the data-flows targets identified from the analysis of high level objectives. The set of potential targets (Target Nodes) is eventually fed into an attack graph generation tool, along with the architectural model of the CPS, to compute exploitation paths eventually leading to a high level threat.

\subsection{Computation of Logical Attack Graphs}

In this work, we use the formalisation of attack graphs introduced by [30] and [29] to model security aspects of a Cyber Physical System. An Attack Graph $\mathcal{A G}$ is defined as a 6-tuple

$$
\mathcal{A G}=\left(V_{r}, V_{p}, V_{d}, E, \mathcal{D}, \mathcal{G}\right)
$$

In this formalism, $V_{r}, V_{p}$ and $V_{d}$ are the three kind of vertices that compose the graph and represent respectively: derivation rules, primitive and derived facts. $\mathcal{D}$ is a set of Descriptions labels associated to each vertex and $\mathcal{G}$ is the last vertex of the graph and objective of the attacker. Edges in the graph are enclosed in set $E$. When multiple edges are input for a derivation node $v \in V_{r}$, these must be read in and with each other. They represent all the conditions the attacker needs to meet to advance a step towards the 
objective. On the other hand, when multiple edges connect different derivation nodes $v_{1}, v_{2} \in V_{r}$ with a derived fact $w \in V_{d}$, these first are read in or with each other, meaning the existence of different paths (exploitation of different node) to reach the same node $w$. An

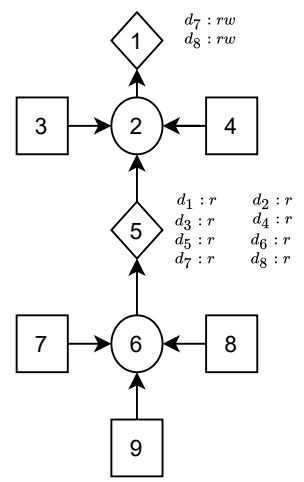

\begin{tabular}{|c|c|}
\hline Label & Description \\
\hline 1 & compromisedAlgorithm(Controller) \\
\hline 2 & exploitVuln (Controller, CVE123, canbus) \\
\hline 3 & vulnExist(Controller, CVE123, canbus) \\
\hline 4 & hacl(Controller, canbus) \\
\hline 5 & canAccess(attacker,canbus) \\
\hline 6 & exploitVuln(DataBase, CVE456, subnet) \\
\hline 7 & vulnExist(Database, CVE456, subnet) \\
\hline 8 & hacl(Database, subnet) \\
\hline 9 & attackerLocated(subnet) \\
\hline
\end{tabular}

Figure 5: Example of Logical Attack Graph built for a lowlevel subset of on-board components of the Communication Based Train Control (CBTC) system. Node 1 is a Target Node and 11 the attack injection point.

example of an Attack Graph built for a low-level subset of on-board components of the Communication Based Train Control (CBTC) system is reported in Figure 5. The AG is rooted in 1 which is the Target Node corresponding to a high level objective previously identified. Vertices $V_{r}, V_{p}$ and $V_{d}$ are respectively drawn as circles, squares and rhomboids. The injection point of the attack (physical attack location) is within the network subnet (Node 9). From there, adversaries have direct access to an onboard database 4 which is on the same network (Node 8). Node 7 shows that the database is affected by a vulnerability CVE 2020-456 the exploitation of which (Node 5) enables the attacker to earn enough privileges to access the Controller Area Network. At this stage, the attacker has gained read permission on data-flows transmitted on this channel. Given the architecture topology, the attacker is now on the same network as the controller. This, along with the fact that the Controller vulnerable to CVE2020-123 is on the same network (Node 4), enables the attacker to run an exploit and upload a malicious payload on the Controller (Node $1 \in \mathcal{G}$ ) which was the Target Node, eventually gaining read and write privileges on $d_{7}, d_{8}$. The attacker is now able to directly tamper with the issued control action. The information provided by the attack graphs is useful for both risk assessment and countermeasure evaluation. From a risk assessment perspective, if a probability of success is associated with each vulnerability (successful exploitation), it is possible to infer the probability of compromise of subsequent components in the attack paths, including the Target nodes [27]. Therefore by knowing the probability of a successful exploitation of the vulnerabilities, it is possible to quantify the risk that a hazardous scenario will be triggered by an attack. On the other hand, countermeasure strategies can be evaluated by dynamically disabling pre-conditions for derivation rules as the attacker moves along the graph.

\footnotetext{
${ }^{4}$ i.e. media server or historian
}

We introduced the core elements of the proposed hazard driven methodology for threat modelling in the context of Cyber Physical Systems. The process comprises three steps and covers the different aspects of the threat analysis, from the definition of high level objectives down to the enumeration of potential target within the CPS deployment. We use tools like MULVAL [30] to compute an Attack Graph given a potential target and the system architecture.

\section{THREAT MODELLING OF A CBTC SYSTEM}

In this Section, we provide an example of the application of our methodology to the System Under Test (SUT). In particular, we show how, given a high level objective it is possible to enumerate architectural components which are potential targets. We then perform a simulation of an attack against selected Target Nodes via Matlab/Simulink, aiming to verify that the high level objective is achieved by adversarial actors that exploit weaknesses and vulnerabilities along the exploitation paths found. For the sake of brevity, we assume the scope of the attacker is restricted to the ground infrastructure and the train $i$, meaning that we do not consider attacks against the other $N-1$ trains in the system. This hypothesis does not limit our application because (1) We assumed that trains are identical to one another. (2) Given the topology of the communication and the kind of infrastructure, the restrictions that we imposed on attacker's scope do not exclude any abuse case. Moreover, since each train only communicates with convoys immediately behind or ahead, the complexity of the model scales linearly with the number of trains. Since our methodology is agnostic to the nature of the system analysed, the example that we bring can be generalised to a wider class of Cyber Physical Systems. To this extent, we simulate different attacks against both the inner control loop and the external one.

\subsection{Disruption of a Service}

In Section 3, a System Theoretic Process Analysis has been carried out for the Communication Train Based Control system and results have been summarised in Table 1. Here, we investigate the following two high-level objectives: (1) Collision between the $i$-th train and the one that precedes (2) Derailment of the $i$-th train.

A Collision event has been labelled as $\mathbf{H 1}$ within the hazard analysis of the System and, from the study of Table 1 (Section 3), it can be caused by unsafe application of Control Actions Brake and Throttle in the following cases:

(1) Brake: Not Applied when Required, Wrong Timing (Applied Late), Wrong Duration (Stops Early).

(2) Throttle: Applied when not required, Wrong Duration (Stops Late).

We also know that Brake $\left(d_{8}^{(i)}\right)$ and Throttle $\left(d_{7}^{(i)}\right)$ are Stream Control Action.

The process proceeds with the analysis of the attack modalities for these two Control Actions. Unsafe application of $d_{8}^{(i)}$ can be caused by direct attacks against the control action through the exploitation of availability and integrity threats on Target Nodes $\theta_{1}, \theta_{2}, \ldots \theta_{K}$ where $\theta_{k} \in \Phi\left(d_{8}^{(i)}\right)$ and $k=1, . ., K$ is the number of architectural components that handle $d_{8}^{(i)}$. These are the Brake 
Actuator, Canbus and Train Controller represented in Figure 4. Furthermore, unsafe application of $d_{8}^{(i)}$ can also be caused via indirect attacks against control action or feedback i.e., the integrity of dataflows in a precedence relationship with that targeted. These are $d_{1}^{(i)}, d_{4}^{(i)}, d_{5}^{(i)}, d_{6}^{(i)}$ of first degree and $d_{0}$ of second degree. Therefore, we add to the list of Target Nodes components $\theta_{1}, \theta_{2}, \ldots \theta_{M}$ where $\theta_{k} \in \Phi\left(d_{1}^{(i)}\right) \cup \Phi\left(d_{4}^{(i)}\right) \cup \Phi\left(d_{5}^{(i)}\right) \cup \Phi\left(d_{6}^{(i)}\right), k=1, . ., M$. These are the Position Sensor, Speed Sensor, Controller of preceding train, Radio, Zone Controller, etc. represented in Figure 4. Finally, we feed the list of Target Nodes into an Attack Graph computation tool such as MulVal to obtain an AG like the one showed in Figure 5. On the other hand, a Derail event (H2) can be caused by unsafe application of Control Actions Brake and Speed Limit (Table 1) in the following cases:

(1) Brake: Not Applied when Required, Wrong Timing (Applied Late), Wrong Duration (Stops Early).

(2) Speed Limit: Applied when not required.

While the analysis of the Brake Control Action is equivalent to the same case for the collision hazard, it is interesting to analyse the unsafe application of the Control Action $d_{1}^{(i)}$ (Speed Limit). In fact, this is an Event Control Action and its unsafe application can be caused by an adversary acquiring Zone Controllers privileges (Figure 4) to write on the Speed Limit database. As for the other cases, we first find all the Target Nodes handling the flow $d_{1}^{(i)}$ and its predecessors and then build a series of Attack Graphs for this high level objective.

\subsection{Mission hijack}

In order to hijack the mission of a CPS, our attacker needs to be familiar with the internal dynamics of the physical process and the control laws, which are described in the behavioural model. Malicious actors must also have significant knowledge of the architectural model to be able to observe the process state and provide control inputs. In other words, they need the capability to read system measurements and factor and provide control signals. .

In order to hijack the mission of the CBTC system, the attacker can compromise different elements of its control structure. A first option is to target the train controller, to gain read privileges on a subset of measurements from sensors. From an analytical perspective, attackers must be able to access enough measurements of the process state (output) so that the Controllability (Observability) matrix of the loop Attacker-Process has full rank [15]. Attackers also need privileges to write the control action. To this extent, we populate the list of Target Nodes with components $\theta_{1}, \theta_{2}, \ldots \theta_{M}$ where $\theta_{k} \in\left\{\Phi\left(d_{5}^{(i)}\right) \cup \Phi\left(d_{6}^{(i)}\right) \cup \Phi\left(d_{7}^{(i)}\right) \cup \Phi\left(d_{8}^{(i)}\right)\right\}, k=1, . ., M$. Moreover, the attacker has also the option for exploiting the distributed structure of the system. Distributed control systems feature a set of processes evolving together according to a predefined synchronisation criteria. Attackers can target a small subset of controllers (or the information exchange between them) to pilot the behaviour of the entire fleet. A small scale example of this scenario is implemented in Figure 6e. By attacking the integrity of the communication between Leader and follower, the latter is brought to synchronise its state (position and speed) with the received, forged, information. As a result, the attacker disrupts the service by increasing inter-train distances, thus reducing the throughput of the whole infrastructure.

\subsection{Attack Simulations}

Given the threats found in Section 6.1 and 6.2, we performed three simulations of attacks against the CPS at control level. Results are shown in Figure 6 and prove that the acquisition of read and write privileges by an attacker on a part of the information data-flow results in the hazard hypothesised at the beginning of the analysis - the high-level objective of an attacker. Figures $6 \mathrm{a}$ and $6 \mathrm{~b}$ show the normal behaviour of the train, controlled via a Communication Based Train (CBTC) in absence of attacks: the convoys' position proceeds over time, and the Follower train always keeps a safe distance of at least thirty meters. The first attack simulation is represented in Figures 6c, 6d and consists of an action of Additive False Data Injection (AFDI) applied at time $t=150$, achievable through the exploitation of any Target Node with write privileges on data-flows $d_{7}^{(i)}$ and $d_{8}^{(i)}$. We leverage the mapping done in Section 4.1 to highlight that, from a control theoretic perspective, the control signal driving the $i$-th train becomes $\hat{u}_{i}^{\text {int }}(t)=u_{i}^{\text {int }}(t)+u_{a}(t)$, where $u_{i}^{\text {int }}$ is the same as defined in equation 1 and $u_{a}$ is the additive action imposed by the attacker. In the particular case of the simulation, although the hazardous scenario has been triggered as the intertrain distance drops below $30 \mathrm{~m}$, the integral action of $u_{i}^{\text {int }}$ partially rejected the attacker's injected signal, avoiding a catastrophic collision: $p_{2}(t)$ does not overlap with $p_{1}(t)$. In Figures 6e and 6f, we reported the results from an attack whose high level objective is to hijack the scope of the mission. In this case, attackers gain read/write privileges on the $d_{4}^{(i)}$ and tamper with distributed information received from the preceding train. From a control perspective (System Theoretic to Behavioural mapping), the position $\hat{p}_{i-1}(t)$ used by the controller of the $i-t h$ train $i$ becomes $\hat{p}_{i-1}(t)=p_{i-1}(t)+p_{a}(t)$, where $p_{i-1}(t)$ is the actual position of $i-1$ and $p_{a}$ the injected false data. Knowing the original control objective $\hat{p}=p_{i}(t)-p_{i-1}(t)$ with $\hat{p}$ the safety distance, the attacker injects $p_{a}>0$ such that the new control objective becomes $\hat{p}+p_{a}=p_{i}(t)-p_{i-1}(t)$. Finally, in Figures $6 \mathrm{~g}$ and $6 \mathrm{~h}$ we show the results of an attack against the Speed Limit. The high level objective of the attacker, in this case, is to disrupt the system by imposing a speed limit that is lower than the original imposed by the system operator. As a result, the attacker successfully slows the follower train.

We used the threat model introduced in Section 5 to enumerate Target Nodes, given high-level assumptions about attacker objectives. Namely, starting from an abuse case, we leveraged STPA analysis to list the information data-flow concerned. Finally, we enumerated Target Nodes participating it and used them as basis to build an attack graph (AG) for the objective.

\section{IMPACT ASSESSMENT}

In the previous Section we leveraged the two mappings found between functional and, respectively, behavioural and architectural model of the CPS to perform threat modelling. In particular, we started from the specification of high level objectives such as Service Disruption and Mission Hijacking and found the information that the attacker needs to access with either read or write permissions. We then found all the architectural components responsible for 


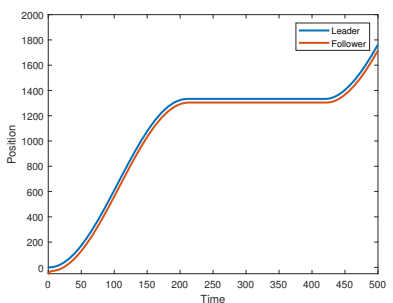

(a)

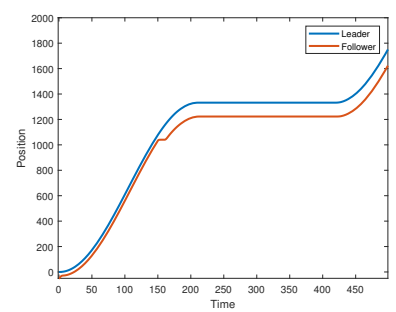

(e)

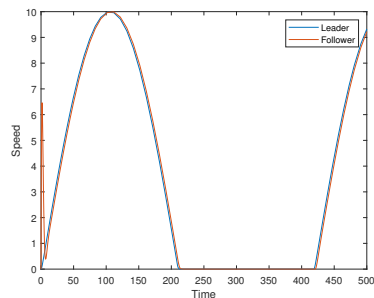

(b)

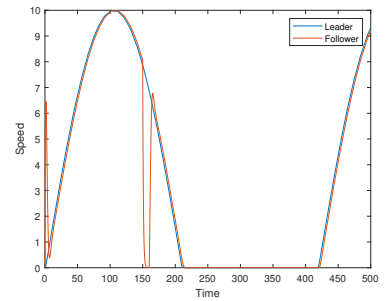

(f)

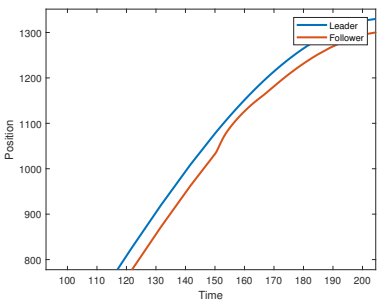

(c)

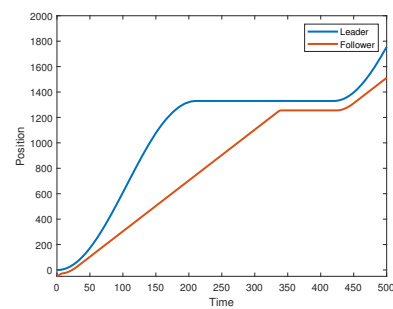

(g)

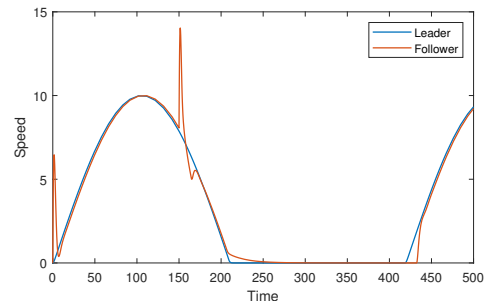

(d)

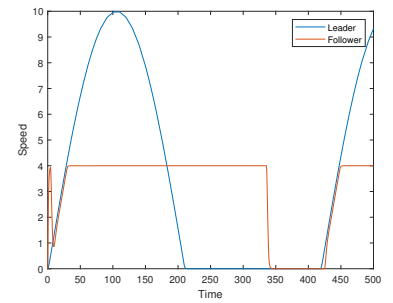

(h)

Figure 6: Four simulations of Attacks have been conducted. (a) and (b) show the evolution of position and speed of Leader and Follower over time in absence of attacks; (c) and (d) show the evolution of position and speed of leader and follower in case of constant AFDI $d_{7}^{(i)}$ and $d_{8}^{(i)}$ starting at $t=150$; (e) and (f) show the evolution of position and speed of leader and follower in case of constant AFDI $d_{4}^{(i)}$ starting at $t=150$; (g) and (h) show the evolution of position and speed of leader and follower in case of attack on Speed Limit of Train $i$.

handling the data-flow (Target Nodes) and built an Attack Graph (AG) for all of them. In this Section, we leverage the existing AG to perform a qualitative and quantitative assessment of the impact of an attack, when the Intrusion Detection System notifies a possible intrusion on a Target Node. The outcomes from this analysis can be used to engineer real-time resilience strategies.

\subsection{Qualitative assessment}

Given an intrusion detected on a Target Node in the system, we leverage the mapping proposed in Section 4.2 to highlight the dataflows impacted by the attack and, consequently, which Feedback and Control Action are exposed and, therefore, which hazardous scenarios could be triggered. With reference to Figures 4 and 5, for instance, an attack detected on the Position Sensor would eventually impact Control Actions Brake and Throttle as they are in a precedence relationship with the sensor's output. To this extent, we know that the system is now exposed to hazards Collision, Derail and Service Disruption (Table 1). At this stage it is impossible to predict the hazard that will actually occur, as the action (i.e. AFDI) of the attacker is not quantified.

\subsection{Quantitative assessment}

The quantitative assessment of the an attack against a Target Node is of utmost importance to engineer strategies to protect the system at design level. This operation is built on the top of qualitative assessment discussed above. In this case we leverage the map between behavioural and system theoretic model to investigate the role played the data-flow in the control model (parameter, process output, controller output, etc.) and the variations that we can allow for it. For example, recall the attack simulation represented in Figure 6e. In this case the new control objective, imposed by the attacker, becomes

$$
\hat{p}+p_{a}(t)=p_{i}(t)-p_{i-1}(t)
$$

where $\hat{p}$ is the safety distance, $p_{a}(t)$ the action of the attacker and $p_{i}(t), p_{i-1}(t)$ respectively the positions of trains $i$ and $i-1$. If the injected value is such that $p_{a}(t)<0$ the distance between the train reduces until a collision verifies. Quantitative assessment techniques can help in deciding the span $p_{a}(t)$. In fact, to ensure business continuity, it could be more convenient to tolerate small perturbations while sanitising architectural components rather than halt or reduce system functionality.

\section{CONCLUSIONS AND FUTURE WORKS}

In this work, we presented a novel methodology to perform hazard driven threat modelling of Cyber Physical Systems. By joining together different representations modelling several aspects of the CPS we are able to evaluate (1) Attack paths that can be monitored in order to improve detection and response to attacks; (2) Qualitative impact to know which system functionalities will be impaired and a range of potential safety violations that can be caused when an attack is detected on a system node; (3) Quantitative impact to allow for finer grain monitoring and tolerance against adversarial perturbations. In this work, we shown the potential to directly map high-level attack goals onto the system architecture, which also denotes the attack surface. High level goals are derived on the basis of requirements that are usually defined at design stage, far before 
the definition of the system architecture itself. The process starts with the identification of attacker capabilities, scope and system level objectives, based on the results of a hazard analysis. We then investigated which data the attacker needs to access and use these findings to enumerate nodes that constitute a potential target for an attack. Finally, we found exploitation paths leading to targets within the architectural model and made use of the computed attack graphs to perform an assessment of attack impact. The main limitation of our work lies in the mapping between the different models of the Cyber Physical System. In fact, while we believe that it is possible to define a set of rules to partially automate the mappting between the architectural and the ST model, this cannot always be easily achieved for large heterogeneous system where components of the CPS are different one from another. Future works include the definition of rules and the design of new approaches to (partially) automate the mapping process. Another minor limitation is related to the assumption that functional, behavioural and architectural models are all available for the analysis in an open and machine readable format. A further interesting direction for future work is to investigate the use of the generated attack graph to synthesise strategies to guarantee business continuity in Cyber Physical Systems under attack. An interesting use case is provided by providing online countermeasure selection by re-configuring the system architecture aiming to disable vulnerability dependencies as the attacker proceeds laterally across the infrastructure.

\section{REFERENCES}

[1] Marshall Abrams and Joe Weiss. 2008. Malicious control system cyber security attack case study-Maroochy Water Services, Australia. McLean, VA: The MITRE Corporation (2008).

[2] Sofia Belikovetsky, Mark Yampolskiy, Jinghui Toh, Jacob Gatlin, and Yuval Elovici. 2017. dr0wned-Cyber-physical attack with additive manufacturing. In 11th \{USENIX\} Workshop on Offensive Technologies (\{WOOT $\}$ 17).

[3] Alvaro A Cardenas, Saurabh Amin, and Shankar Sastry. 2008. Secure control: Towards survivable cyber-physical systems. In 2008 The 28th International Conference on Distributed Computing Systems Workshops. IEEE, 495-500.

[4] Defense Use Case. 2016. Analysis of the cyber attack on the Ukrainian power grid. Electricity Information Sharing and Analysis Center (E-ISAC) (2016).

[5] John H Castellanos, Martín Ochoa, and Jianying Zhou. 2018. Finding dependencies between cyber-physical domains for security testing of industrial control systems. In Proceedings of the 34th Annual Computer Security Applications Conference. 582-594.

[6] Crowdstrike. 2020. 2020 Crowdstrike Global Threat Report. Report (2020).

[7] Alessandro Di Pinto, Younes Dragoni, and Andrea Carcano. 2018. TRITON: The first ICS cyber attack on safety instrument systems. In Proc. Black Hat USA. 1-26.

[8] Robert Ellison, John Hudak, Rick Kazman, Carol Woody, and Allen Householder 2015. Extending aadl for security design assurance of cyber physical systems. Technical Report. CARNEGIE-MELLON UNIV PITTSBURGH PA PITTSBURGH United States.

[9] Clifton A Ericson et al. 2015. Hazard analysis techniques for system safety. John Wiley \& Sons.

[10] Song Fang, Karl Henrik Johansson, Mikael Skoglund, Henrik Sandberg, and Hideaki Ishii. 2019. Two-way coding in control systems under injection attacks from attack detection to attack correction. In Proceedings of the 10th ACM/IEEE International Conference on Cyber-Physical Systems. ACM, 141-150.

[11] Peter H Feiler, David P Gluch, and John J Hudak. 2006. The architecture analysis \& design language (AADL): An introduction. Technical Report. Carnegie-Mellon Univ Pittsburgh PA Software Engineering Inst

[12] Ivo Friedberg, Kieran McLaughlin, Paul Smith, David Laverty, and Sakir Sezer. 2017. STPA-SafeSec: Safety and security analysis for cyber-physical systems. Journal of Information Security and Applications 34 (2017), 183-196.

[13] Kian Gheitasi, Mohsen Ghaderi, and Walter Lucia. 2019. A Novel Networked Control Scheme with Safety Guarantees for Detection and Mitigation of CyberAttacks. In 2019 18th European Control Conference (ECC). IEEE, 1449-1454.

[14] Jairo Giraldo and Alvaro A Cardenas. 2019. Moving target defense for attack mitigation in multi-vehicle systems. In Proactive and Dynamic Network Defense. Springer, 163-190.
[15] Adam Hahn, Roshan K Thomas, Ivan Lozano, and Alvaro Cardenas. 2015. A multi-layered and kill-chain based security analysis framework for cyber-physical systems. International fournal of Critical Infrastructure Protection 11 (2015), 39-50.

[16] Zhongyuan Hau, John H Castellanos, and Jianying Zhou. 2019. Evaluating Cascading Effects of Attacks on Resilience of Industrial Control Systems: A Design-Centric Modeling Approach. arXiv preprint arXiv:1905.03156 (2019).

[17] Bing Huang, Alvaro A Cardenas, and Ross Baldick. 2019. Not everything is dark and gloomy: Power grid protections against IoT demand attacks. In 28th \{USENIX\} Security Symposium ( $\{$ USENIX\} Security 19). 1115-1132.

[18] Kip Johnson. 2017. Extending Systems-Theoretic Safety Analyses for Coordination. STAMP Workshop '17 (2017).

[19] Sahand Hadizadeh Kafash, Jairo Giraldo, Carlos Murguia, Alvaro A Cardenas, and Justin Ruths. 2018. Constraining attacker capabilities through actuator saturation. In 2018 Annual American Control Conference (ACC). IEEE, 986-991.

[20] Ralph Langner. 2011. Stuxnet: Dissecting a cyberwarfare weapon. IEEE Security \& Privacy 9, 3 (2011), 49-51.

[21] Nancy Leveson. 2011. Engineering a safer world: Systems thinking applied to safety. MIT press.

[22] N Leveson and J Thomas. 2019. STPA Handbook (2018).

[23] Stefano Longari, Andrea Cannizzo, Michele Carminati, and Stefano Zanero. 2019. A Secure-by-Design Framework for Automotive On-board Network Risk Analysis. In 2019 IEEE Vehicular Networking Conference (VNC). IEEE, 1-8.

[24] Charlie Miller and Chris Valasek. 2015. Remote exploitation of an unaltered passenger vehicle. Black Hat USA 2015 (2015), 91.

[25] Yilin Mo and Bruno Sinopoli. 2015. On the performance degradation of cyberphysical systems under stealthy integrity attacks. IEEE Trans. Automat. Control 61, 9 (2015), 2618-2624.

[26] Naeem Muhammad, Yves Vandewoude, Yolande Berbers, and Sjir Van Loo. 2009. Modelling composite end-to-end flows with AADL. In Proceedings of the workshop on the definition, evaluation, and exploitation of modelling and computing standards for Real-Time Embedded Systems. 1-3.

[27] Luis Muñoz-González, Daniele Sgandurra, Martín Barrère, and Emil C Lupu. 2017. Exact inference techniques for the analysis of Bayesian attack graphs. IEEE Transactions on Dependable and Secure Computing 16, 2 (2017), 231-244.

[28] Arash Nourian and Stuart Madnick. 2015. A systems theoretic approach to the security threats in cyber physical systems applied to stuxnet. IEEE Transactions on Dependable and Secure Computing 15, 1 (2015), 2-13.

[29] Xinming Ou, Wayne F Boyer, and Miles A McQueen. 2006. A scalable approach to attack graph generation. In Proceedings of the 13th ACM conference on Computer and communications security. 336-345.

[30] Xinming Ou, Sudhakar Govindavajhala, and Andrew W Appel. 2005. MulVAL: A Logic-based Network Security Analyzer.. In USENIX security symposium, Vol. 8. Baltimore, MD, 113-128.

[31] Antonio Roque, Kevin B Bush, and Christopher Degni. 2016. Security is about control: insights from cybernetics. In Proceedings of the Symposium and Bootcamp on the Science of Security. ACM, 17-24.

[32] Alastair Ruddle, David Ward, Benjamin Weyl, Sabir Idrees, Yves Roudier, Michael Friedewald, Timo Leimbach, Andreas Fuchs, Sigrid Gürgens, Olaf Henniger, et al. 2009. Deliverable D2. 3: Security requirements for automotive on-board networks based on dark-side scenarios. In EVITA project.

[33] Jill Slay and Michael Miller. 2007. Lessons learned from the maroochy water breach. In International Conference on Critical Infrastructure Protection. Springer, 73-82.

[34] Joe Slowik. 2018. Anatomy of an Attack: Detecting and Defeating CRASHOVERRIDE. VB2018, October (2018).

[35] Yonglei Tao and Chenho Kung. 1991. Formal definition and verification of data flow diagrams. Fournal of Systems and Software 16, 1 (1991), 29-36.

[36] André Teixeira, Henrik Sandberg, and Karl H Johansson. 2010. Networked control systems under cyber attacks with applications to power networks. In Proceedings of the 2010 American Control Conference. IEEE, 3690-3696.

[37] John P Thomas IV. 2013. Extending and automating a systems-theoretic hazard analysis for requirements generation and analysis. Ph.D. Dissertation. Massachusetts Institute of Technology.

[38] Tony UcedaVelez. 2012. Real World Threat ModelingUsing the PASTA Methodology. https://owasp.org/www-pdf-archive/AppSecEU2012_PASTA.pdf

[39] William E Young. 2014. STPA-SEC for cyber security mission assurance. Eng Syst. Div. Syst. Eng. Res. Lab (2014) 